\title{
Urinary iodine in early pregnancy is associated with subclinical hypothyroidism in Tianjin, China: an observational study
}

Kunling Wang ${ }^{1 \dagger}$, Jie Zhang ${ }^{2 \dagger}$, Fengao Li', Wanqi Zhang ${ }^{3}$, Hao Wang ${ }^{1}$, Li Ding ${ }^{1}$, Yaxin Liu', Laixiang Lin Shuang Zhang ${ }^{5}$ and Mei Zhu ${ }^{1 *}$

\begin{abstract}
Background: Subclinical hypothyroidism (SH) is associated with adverse obstetric outcomes and neurodevelopment disorders. Both iodine deficiency and excess are associated with $\mathrm{SH}$; however, few data regarding iodine nutrition status of pregnant women with SH are available. This study aimed to clarify whether iodine deficiency or excess is associated with SH, especially, when test results for anti-thyroid autoantibodies are negative.
\end{abstract}

Methods: A total of 115 women with SH and 104 women with euthyroidism (EH) in early pregnancy in Tianjin, China were investigated, and their serum thyroid-stimulating hormone, free thyroxine, free triiodothyronine, anti-thyroid peroxidase antibody (TPOAb), anti-thyroid globulin antibody (TGAb), urinary iodine (UIC), and urinary creatinine (UCr) concentrations were measured. Thyroid ultrasonography was performed to determine thyroid echogenicity and volume. The UIC, UIC/UCr ratio, prevalence of TPOAb and TGAb positivity, and thyroid gland volume were compared between the EH and SH groups. UIC and ultrasonographic features were analysed in subjects in the SH group who were negative for TPOAb and TGAb.

Results: Median UIC of SH $(154.0 \mu \mathrm{g} / \mathrm{L})$ and EH $(150.1 \mu \mathrm{g} / \mathrm{L})$ met the World Health Organization criterion for iodine sufficiency in pregnant women. Neither UIC nor the UIC/UCr ratio differed significantly between groups. The prevalence of TPOAb and TGAb positivity in the SH group was significantly higher than that in the EH group $(P<0.01)$. The percentage of subjects with $\mathrm{UIC} \geq 250 \mu \mathrm{g} / \mathrm{L}$ in the $\mathrm{SH}$ group was significantly higher than that in the EH group $(p=0.004)$. The percentage of subjects negative for autoantibodies and $\mathrm{UIC} \geq 250 \mu \mathrm{g} / \mathrm{L}$ in the SH group tended to be higher than that in subjects in the EH group negative for autoantibodies, but the difference was not statistically significant ( $p=0.025$, adjusted test level $a=0.0167$ ). Eight of 18 subjects in the SH group with negative results for TPOAb and TGAb were diagnosed with Hashimoto thyroiditis by means of thyroid ultrasonography.

Conclusions: Women in early pregnancy with SH in Tianjin were iodine sufficient, but still at risk of iodine deficiency as pregnancy progressed. UIC $\geq 250 \mu \mathrm{g} / \mathrm{L}$ was associated with increased risk of SH. Serological negative autoimmune thyroiditis and UIC $\geq 250 \mathrm{\mu g} / \mathrm{L}$ may play a role in pathogenesis of $\mathrm{SH}$ cases with negative results for autoantibodies.

Keywords: Early pregnancy, Subclinical hypothyroidism, Urine iodine concentration, Anti-thyroid peroxidase antibody (TPOAb), Anti-thyroid globulin antibody (TPOAb), Idiopathic subclinical hypothyroidism

\footnotetext{
* Correspondence: meichuqin@163.com

${ }^{\dagger}$ Equal contributors

${ }^{1}$ Department of Endocrinology and Metabolism, Tianjin Medical University

General Hospital, No154 Anshan Road, Heping District, Tianjin 300052, China

Full list of author information is available at the end of the article
} 


\section{Background}

Hypothyroidism is a common comorbidity of pregnancy and can be further categorised as clinical or subclinical hypothyroidism $(\mathrm{SH})$ according to reduced or normal free thyroxine $\left(\mathrm{FT}_{4}\right)$ levels. Clinical hypothyroidism can result in retarded foetal growth, mental development disorders, and adverse obstetric outcomes. Mounting clinical evidence suggests that $\mathrm{SH}$ contributes to an increased rate of obstetric complications and adverse birth outcomes including gestational diabetes, gestational hypertension, miscarriage, premature birth, small-forgestational-age babies, placental abruption, and postpartum haemorrhage [1-4]. It is estimated that the prevalence of $\mathrm{SH}$ in pregnancy is $2.0-2.5 \%$ [5] worldwide and $4 \%$ [6] in China.

Iodine is the most important trace element for thyroid hormone synthesis, and both iodine deficiency and iodine excess are associated with $\mathrm{SH}[7,8]$. There was widespread iodine deficiency in China until universal salt iodisation in 1995. Since then, the prevalence of iodine deficiency diseases has decreased remarkably, while the prevalence of autoimmune thyroiditis and $\mathrm{SH}$ increased [9]. Pregnant women are vulnerable to iodine deficiency, and some cross-sectional surveys in different provinces and areas of China have showed iodine deficiency in pregnancy $[10,11]$. However, few data regarding iodine nutrition status in pregnant women with $\mathrm{SH}$ are available. An epidemic investigation in Tianjin, a northern coastal city in China that is abundant in marine products, revealed iodine sufficiency [12]. To determine the iodine nutrition status and the prevalence of positivity for anti-thyroid peroxidase antibody (TPOAb) and antithyroid globulin antibody (TGAb) in early pregnancy with $\mathrm{SH}$ in Tianjin, this study evaluated the urinary iodine concentration (UIC), TPOAb and TGAb positivity, and ultrasonographic features of the thyroid gland in early pregnancy in relation to $\mathrm{SH}$, considering evidence regarding iodine supplementation, anti-thyroid autoantibody screening, and proper intervention.

\section{Methods}

\section{Subjects}

Women in early pregnancy diagnosed as $\mathrm{SH}$ in the outpatient clinic of the endocrinology department of Tianjin Medical University General Hospital from September 2014 to October 2016 were included in the study. The control group comprised those in early pregnancy diagnosed as euthyroidism $(\mathrm{EH})$ in the same hospital. Gestational age was determined from foetal B mode ultrasonography and the time of the last menstrual period. All subjects were local residents and had lived in Tianjin more than 5 years. Women with history of thyroid diseases or other comorbidities and women who took thyroid medications, anti-thyroid drugs, iodine-containing medications or supplements, or iodinated contrast agents (e.g. amiodarone, cydiodine buccal tablets, intravenous contrast agents, and iodine-containing nutrient supplements) within the past 3 months were excluded from the study.

The study was conducted in accordance with the principles of good clinical practice and the Declaration of Helsinki. The study protocol was approved by The Ethics Committee of Tianjin Medical University General Hospital, and written informed consent was obtained from all participants.

\section{Sample collection}

Five millilitres fasting venous blood, without coagulation, was drawn from all participants. Serum was separated within $6 \mathrm{~h}$ and stored at $-80{ }^{\circ} \mathrm{C}$. Ten millilitres of fasting urine were collected in specialised containers and stored at $-80{ }^{\circ} \mathrm{C}$ for testing UIC and urinary creatinine (UCr) concentration.

\section{Testing of thyroid function}

Serum thyroid-stimulating hormone (TSH), free thyroxine $\left(\mathrm{FT}_{4}\right)$, free triiodothyronine $\left(\mathrm{FT}_{3}\right)$, thyroid peroxidase antibody (TPOAb), and thyroid globulin antibody (TGAb) levels were all tested using a chemiluminescence immunoassay test kit from Siemens, Germany. Quality control was performed for all test kits. The intra- and inter-assay coefficients of variation $(n=20)$ in our laboratory were, respectively, 2.7 and $4.6 \%$ for $\mathrm{FT}_{3}, 3.2$ and $4.9 \%$ for $\mathrm{FT}_{4}, 2.6$ and $4.6 \%$ for $\mathrm{TSH}, 7.2$ and $11.2 \%$ for TPOAb, and 7.9 and $12.3 \%$ for TGAb.

\section{Testing of UIC and $\mathrm{UCr}$}

UIC was estimated using $\mathrm{As}^{3+}-\mathrm{Ce}^{4+}$ catalytic spectrophotometry (WS/T-2006), a national method developed by China's Ministry of Health. To exclude the impact of the volume and level of urine concentration on UIC, $\mathrm{UCr}$ was tested along with urinary iodine, and the UIC/ $\mathrm{UCr}$ ratio was calculated.

\section{Diagnostic criteria}

All diagnoses were made according to specified reference values for gestational thyroid function as recommended by the guidelines for the diagnosis and treatment of gestational and postpartum thyroid diseases in China proposed in 2012 [13]. Reference values for $\mathrm{TSH}, \mathrm{FT}_{4}, \mathrm{TPOAb}$, and TGAb were $0.13-3.93 \mathrm{mIU} / \mathrm{L}$, 12.00-23.34 pmol/L, 0-35 IU/L, and 0-20 IU/L, respectively. Participants were divided into $\mathrm{EH}$ and subclinical hypothyroidism ( $\mathrm{SH}$ ) groups according to $\mathrm{TSH}$ and $\mathrm{FT}_{4}$ levels. Women with both normal TSH and $\mathrm{FT}_{4}$ were included in the former, while those with TSH levels higher than the upper reference limit and normal $\mathrm{FT}_{4}$ values were included in the latter group. 


\section{Gestational iodine nutritional standards}

Iodine status was classified according to the World Health Organization (WHO) criteria for pregnant women [14]. Median UIC of $<20 \mu \mathrm{g} / \mathrm{L}, 20-50 \mu \mathrm{g} / \mathrm{L}, 51-149 \mu \mathrm{g} / \mathrm{L}, 150$ $249 \mu \mathrm{g} / \mathrm{L}, 250-499 \mu \mathrm{g} / \mathrm{L}$, and $\geq 500 \mu \mathrm{g} / \mathrm{L}$ corresponded to severe, moderate, and mild iodine deficiency, and adequate, more-than-adequate, and excessive iodine intake, respectively.

\section{Ultrasonography}

Thyroid ultrasonography was performed by the same experienced physician using commercially available highdefinition colour-Doppler ultrasound equipment (HD11, Philips Healthcare, Netherlands) equipped with a 7.5-12 $\mathrm{MHz}$ high frequency linear transducer. Patients were examined in a supine position with their neck hyperextended in accordance with a standard sonographic protocol. The volume of each lobe was calculated separately, using a formula for elliptical shape volume $[(\pi /$ $6) \times$ length $\times$ width $\times$ depth]. The thyroid volume was a sum of the volumes of both lobes [15].

\section{Statistical analysis}

Data were analysed using SPSS 19.0 software (IBM, US). Normally distributed data (age and gestational weeks) were reported as mean \pm standard deviation, and skewed data $\left(\mathrm{FT}_{3}, \mathrm{FT}_{4}, \mathrm{TSH}, \mathrm{UIC}\right.$, and $\left.\mathrm{UIC} / \mathrm{UCr}\right)$ were presented as median and interquartile range $\left(25^{\text {th }}-75^{\text {th }}\right.$ percentiles). Two independent-sample $t$-tests were used to compare age and gestational weeks, and Wilcoxon rank sum tests were used to compare $\mathrm{FT}_{3}, \mathrm{FT}_{4}, \mathrm{TSH}, \mathrm{UIC}$, and $\mathrm{UIC} / \mathrm{UCr}$ between the two groups. The chi-squared test or Fisher's exact test was adopted for frequency comparison. Spearman rank bivariate correlation was adopted for correlation analysis. Two-tailed cut-offs were used, and a $P$ value $<0.05$ was considered statistically significant.

\section{Results}

\section{Demographic features of patients}

A total of 219 women in early pregnancy (average $10.1 \pm$ 2.2 weeks) with an average age of $28.3 \pm 3.1$ years were enrolled in the study. One hundred four subjects in the EH group were aged $28.0 \pm 3.0$ years and had gestated for $10.1 \pm 2.3$ weeks, while 115 subjects in the SH group were aged $28.6 \pm 3.2$ years and had gestated for $10.2 \pm 2.0$ weeks. There were no significant differences between age and gestational week between the two groups (Table 1).

lodine nutritional status in early pregnancy with SH in Tianjin The median (range) UIC in the $\mathrm{SH}$ and $\mathrm{EH}$ groups was 154.0(93.1-243.1) $\mu \mathrm{g} / \mathrm{L}$ and 150.1(103.5-187.5) $\mu \mathrm{g} / \mathrm{L}$, respectively, with no significant difference between the groups $(\mathrm{Z}=-1.198, p=0.231)$. The median UIC/UCr ratio in the SH and EH groups was 140.7(98.1-226.4) $\mu \mathrm{g} / \mathrm{g}$ and $131.7(82.8-183.9) \mu \mathrm{g} / \mathrm{g}$, respectively, and was not significantly different between the groups $(\mathrm{Z}=-1.465, p$ $=0.143$ ) (Table 1). Among those in the $\mathrm{SH}$ group, the distribution by $\mathrm{UIC} \leq 50 \mu \mathrm{g} / \mathrm{L}, \quad 51-149 \mu \mathrm{g} / \mathrm{L}, \quad 150$ $249 \mu \mathrm{g} / \mathrm{L}$ and $\geq 250 \mu \mathrm{g} / \mathrm{L}$ levels were 6.0, 43.5, 26.2, and $24.3 \%$, respectively, while those in the $\mathrm{EH}$ group were $5.8,43.3,41.3$, and $9.6 \%$ respectively. There was a significant difference in the percent distributions between the two groups $\left(x^{2}=10.656, p=0.014\right)$. The percentage of those with UIC $\geq 250 \mu \mathrm{g} / \mathrm{L}$ in the SH group was significantly higher than that in the EH group $\left(\chi^{2}=8.265, p=\right.$ 0.004) (Table 2, Fig. 1).

\section{Prevalence of TPOAb and TGAb and its relationship with thyroid function}

The prevalence of TPOAb and TGAb in the $\mathrm{SH}$ group was significantly higher than that in the $\mathrm{EH}$ group (79.1\% vs $56.7 \%, \chi^{2}=12.697, p=0.000$ for TPOAb and $69.6 \%$ vs $40.4 \%, \chi^{2}=18.847, p=0.000$ for TGAb). $\mathrm{FT}_{4}$ level in the $\mathrm{SH}$ group was significantly lower than that in the $\mathrm{EH}$ group $(\mathrm{Z}=-3.157, p=0.002)$, however $\mathrm{FT}_{3}$ and thyroid volume did not differ significantly between the two groups. There was no significant relationship between TSH or $\mathrm{FT}_{4}$ and TPOAb level in women in early pregnancy $(p>0.05)$.

\section{UIC and ultrasonographic features of subjects in the SH} group with negative TPOAb and TGAb

Among subjects in the $\mathrm{SH}$ group, $15.7 \%$ were negative for both TPOAb and TGAb. In the SH group, there were no significant differences in $\mathrm{TSH}$ and $\mathrm{FT}_{4}$ between the autoantibody-negative and -positive subgroups ( $p=$ 0.706 for TSH and $p=0.122$ for $\mathrm{FT}_{4}$ ). To explore the possible aetiology of $\mathrm{SH}$ in subjects with negative antithyroid autoantibodies, we analysed UIC and ultrasonographic features of the subjects. The distribution frequencies in the autoantibody-negative $\mathrm{SH}$ group among those with UIC $<150 \mu \mathrm{g} / \mathrm{L}, 150-249 \mu \mathrm{g} / \mathrm{L}$ and $\geq 250 \mu \mathrm{g} /$ L were $38.9 \%$ (7/18), 33.3\% (6/18), and 27.8\% (5/18), respectively, while those in the autoantibody-negative $\mathrm{EH}$ group were $50.0 \%(20 / 40), 45.0 \%$ (18/40), and 5.0\% (2/ 40), respectively. The distribution frequencies tended to be different between the two groups, but the difference was not statistically significant $(p=0.066)$ (Table 3$)$. The percentage of those with more-than-adequate and excessive iodine in the autoantibody-negative $\mathrm{SH}$ group tended to be higher than that in the autoantibodynegative EH group, but the difference was not statistically significant ( $p=0.025$, adjusted test level $\alpha=0.0167$ ) (Table 3). In the autoantibody-negative SH group, eight of 18 presented as diffuse hypoechoic and heterogeneous echo texture by ultrasonography, and were thereby diagnosed as Hashimoto thyroiditis. 


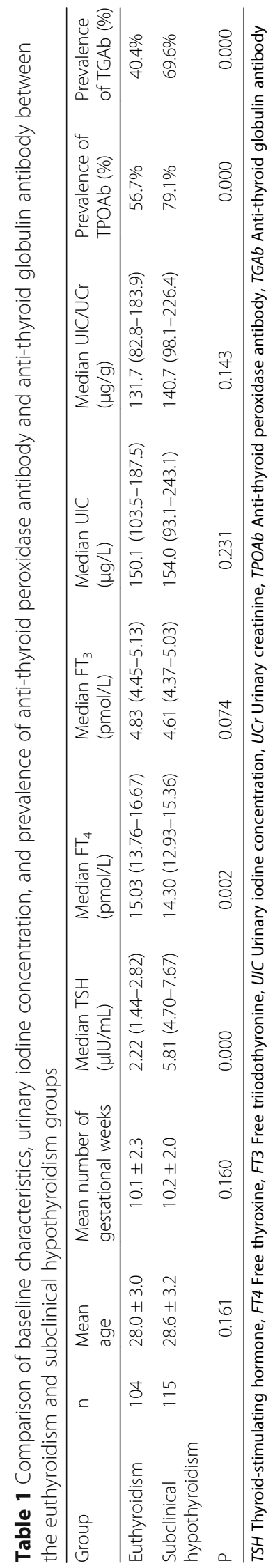


Table 2 Comparison of urinary iodine concentration (UIC) frequency distribution between the euthyroidism and subclinical hypothyroidism groups

\begin{tabular}{llllll}
\hline Group & \multicolumn{4}{l}{ UIC $(\mu \mathrm{g} / \mathrm{L})$ frequency distribution } & Total \\
\cline { 2 - 5 } & $\leq 50$ & $51-149$ & $150-249$ & $\geq 250$ & \\
\hline Euthyroidism & 6 & 45 & 43 & 10 & 104 \\
Subclinical hypothyroidism & 7 & 50 & 30 & 28 & 115 \\
Total & 13 & 95 & 73 & 38 & 219 \\
$P$ & 0.921 & 0.975 & 0.017 & 0.004 & 0.014 \\
\hline
\end{tabular}

The relationship between UIC and thyroid function in early pregnancy

UIC was not significantly correlated with $\mathrm{FT}_{3}, \mathrm{FT}_{4}$, and TSH levels in early pregnancy $(p>0.05)$.

\section{Discussion}

Demand for iodine as a nutrient increases in pregnancy to support a series of physiological changes [16, 17], including increased iodine clearance as a consequence of increased renal blood flow and glomerular filtration, transplacental iodine transfer from mother to foetus, increased demand for thyroid hormone due to maternal hormone transplacental transfer to foetus, and increased combined thyroid hormone due to increased thyroid hormone-binding globulin production in response to higher oestrogen levels. Severe iodine deficiency in pregnancy can lead to maternal hypothyroidism, impairing neurological development of the foetus [18]. Moreover, even mild and moderate iodine deficiency in early pregnancy can impair the final intelligence and scholastic ability of progeny $[19,20]$. On the other hand, excessive iodine intake may be detrimental to pregnant women and increase their risk for developing hypothyroidism and autoimmune thyroiditis [21]. Investigations such as the current study that investigate iodine nutrition status
Table 3 Comparison of urinary iodine concentration (UIC) frequency distribution between the euthyroidism and subclinical hypothyroidism groups with negative anti-thyroid autoantibodies

\begin{tabular}{lllll}
\hline Group & \multicolumn{2}{l}{ UIC $(\mu \mathrm{g} / \mathrm{L})$ frequency } & distribution & Total \\
\cline { 2 - 4 } & $<150$ & $150-249$ & $\geq 250$ & \\
\hline Euthyroidism & 20 & 18 & 2 & 40 \\
Subclinical hypothyroidism & 7 & 6 & 5 & 18 \\
Total & 27 & 24 & 7 & 58 \\
P & 0.433 & 0.404 & 0.025 & 0.066 \\
\hline
\end{tabular}

in women with $\mathrm{SH}$ in early pregnancy may help to provide a basis for proper iodine intake guide.

As $>90 \%$ of ingested iodine is ultimately excreted in the urine, UIC is a good indicator of iodine nutritional status, and median UIC from a large sample in a previous study has been widely used as a biomarker of population iodine intake [22]. WHO recommends an optimum median UIC of $150-249 \mu \mathrm{g} / \mathrm{L}$ for pregnant women [14]. In the current study, median UIC in early pregnant women with subclinical hypothyroidism and euthyroidism was 154.0(93.1243.1) and 150.1(103.5-187.5) $\mu \mathrm{g} / \mathrm{L}$, respectively, both of which are at the lower limit of the iodine sufficiency range. In contrast, results of iodine nutrition status surveys in different geographical regions of China have produced disparate results. In Henan province, an inland area, median UIC in pregnancy was $198 \mu \mathrm{g} / \mathrm{L}$, and the iodine nutrition was adequate [23], while in Zhejiang province, a coastal area, median UIC in pregnancy was $130 \mu \mathrm{g} / \mathrm{L}$, and the iodine nutrition was deficient [11]. In Shijiazhuang province, median UIC declined as gestational age increased, and the iodine nutrition status shifted from iodine sufficiency in the first trimester to deficiency in the second and third trimesters of pregnancy [24]. The present study showed that iodine nutrition status in early pregnancy in Tianjin was adequate, but that median UIC was at the lower limit; in

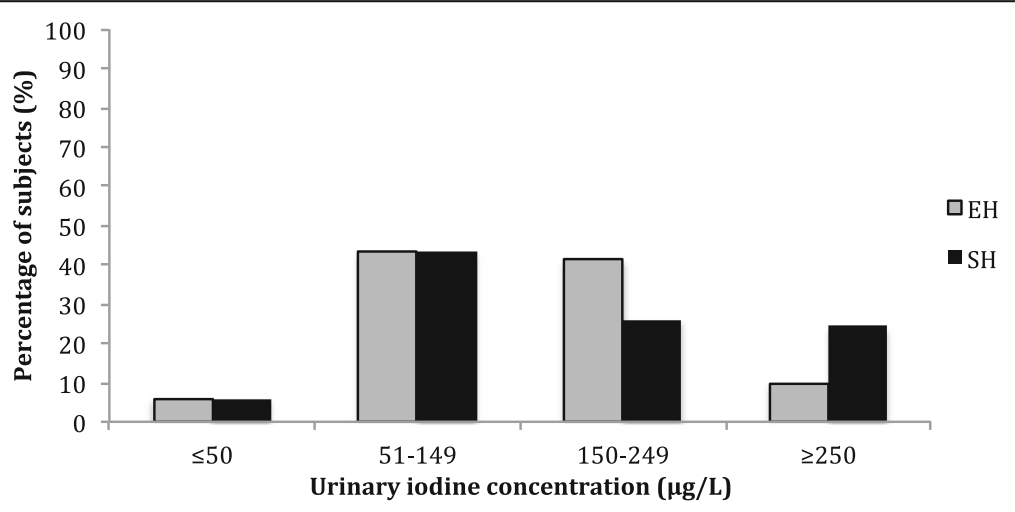

Fig. 1 Urinary iodine concentration (UIC) distribution among early pregnant women with euthyroidism and subclinical hypothyroidism in Tianjin. The percentage distribution was significantly different between the euthyroidism $(E H)$ and subclinical hypothyroidism $(\mathrm{SH}) \mathrm{groups}(\mathrm{X} 2=10.656, p=0.014)$. The percentage of those with UIC $\geq 250 \mu \mathrm{g} / \mathrm{L}$ in the SH group was significantly higher than that in the EH group ( $X 2=8.265, p=0.004)$ 
addition, at mid-gestation, the foetal thyroid gland begins to produce thyroid hormone, leading to transplacental iodine transfer, further exacerbating iodine deprivation of the mother; accordingly, pregnant women in Tianjin are likely still at risk of iodine deficiency as the pregnancy progresses. According to WHO, pregnant women should have an iodine intake of at least $250 \mu \mathrm{g}$ daily [14]. Since the iodine content of edible salt in Tianjin is $30 \mathrm{mg} / \mathrm{kg}$ (permitted range $\pm 30 \%$ ) [25] and the average salt consumption is inadequate at $6 \mathrm{~g} / \mathrm{d}$ (the average reported consumption), pregnant women in Tianjin require additional iodine intake from other sources.

The current study showed that the percentage of those with more-than-adequate and excessive iodine in the $\mathrm{SH}$ group was significantly higher than that in the $\mathrm{EH}$ group. These findings indicate that iodine excess, rather than iodine deficiency, is associated with $\mathrm{SH}$ in early pregnancy in Tianjin. In most cases, excessive iodine intake may inhibit thyroid hormone synthesis and secretion to protect the body from thyrotoxicosis, the socalled acute Wolff-Chaikoff effect [26], which is typically transient and lasts only 24-48 h. However, some vulnerable individuals with underlying thyroid abnormalities might fail to escape from the Wolff-Chaikoff effect, resulting in iodine-induced hypothyroidism [27]. Secondly, iodine excess itself can trigger autoimmune thyroiditis in some genetically susceptible individuals and lead to hypothyroidism. A study conducted by Sang et al. revealed that excessive iodine intake during late pregnancy might lead to maternal thyroid dysfunction, particularly SH [21]. A cross-sectional study of 7190 women in early pregnancy in China also showed that $\mathrm{UIC} \geq 250 \mu \mathrm{g} / \mathrm{L}$ is associated with a significantly high risk of $\mathrm{SH}$ and that $\mathrm{UIC} \geq 500 \mu \mathrm{g} / \mathrm{L}$ is associated with a high risk of isolated hypothyroxinemia [8]. Therefore, pregnant women with $\mathrm{SH}$ should be instructed to consume an appropriate amount of iodine to avoid excessive iodine intake.

The prevalence of TPOAb (79.1\%) and TGAb (69.6\%) positivity in early pregnancy with $\mathrm{SH}$ was significantly higher than that in subjects with euthyroidism, suggesting that autoimmune thyroiditis is the major cause of $\mathrm{SH}$ in early pregnancy in Tianjin. Additionally, TPOAb and TGAb positivity is a risk factor for postpartum thyroiditis [28] and thus deserves particular attention in pregnant women.

Although $15.7 \%$ of subjects in the $\mathrm{SH}$ group were both TPOAb- and TGAb-negative, the serum TSH and $\mathrm{FT}_{4}$ level was not significantly different between the autoantibodynegative and -positive subgroups. Eight of 18 subjects in the $\mathrm{SH}$ group with negative thyroid antibodies were diagnosed as autoimmune thyroiditis by means of thyroid ultrasonography. Rago has confirmed the value of thyroid ultrasonography in detecting autoimmune thyroiditis in cases with negative thyroid antibodies [29]. Therefore, SH with negative anti-thyroid autoantibodies in early pregnancy in the present study might partly be attributable to serological negative autoimmune thyroiditis. Moreover, there tended to be a greater percentage of those with morethan-adequate and excessive iodine in the autoantibodynegative $\mathrm{SH}$ group than that in the autoantibody-negative EH group, therefore more-than-adequate and excessive iodine, rather than iodine deficiency, might be associated with $\mathrm{SH}$ in those with negative thyroid autoantibodies. However due to the small sample size, these findings need to be further investigated.

There were remaining ten subjects both negative for thyroid antibodies and ultrasonography, in the literature, such cases are referred to as "idiopathic subclinical hypothyroidism" [30] or "non-autoimmune subclinical hypothyroidism" [31]. Recently, idiopathic SH was reported to be associated with TSH resistance due to TSH receptor mutations [31]. Furthermore, obesity and overweight are related to elevated TSH level [32], but the contribution of elevated TSH level to $\mathrm{SH}$ in pregnancy remains unclear and needs to be investigated.

There are some limitations in our study. Firstly, the incidence of $\mathrm{SH}$ in pregnancy has been reported to be 2$4 \%$ [6], and some pregnant women do not undergo thyroid function screening in early pregnancy. To achieve a sample size of approximately 100, we screened 4600 women in early pregnancy, thus there was a small sample size. Secondly, the spot UIC used to assess iodine nutrition status of the subjects only reflects recent iodine intake and varies according to extent of hydration, thus limiting its usefulness as a screening tool.

\section{Conclusions}

In summary, the current results show that women with $\mathrm{SH}$ in early pregnancy in Tianjin were iodine sufficient, but remained at risk of iodine deficiency as pregnancy progressed. Moreover, more-than-adequate and excess iodine $(\mathrm{UIC} \geq 250 \mu \mathrm{g} / \mathrm{L})$ were associated with $\mathrm{SH}$. In addition, serological negative autoimmune thyroiditis and $\mathrm{UIC} \geq$ $250 \mu \mathrm{g} / \mathrm{L}$ may play a role in pathogenesis of $\mathrm{SH}$ with negative autoantibodies. However, owing to the small sample size and limited usefulness of spot UIC, a larger sample study is needed to confirm the results.

\section{Abbreviations}

$\mathrm{EH}$ : Euthyroidism; $\mathrm{FT}_{3}$ : Free triiodothyronine; $\mathrm{FT}_{4}$ : Free thyroxine; $\mathrm{SH}$ : Subclinical hypothyroidism; TGAb: Anti-thyroid globulin antibody; TPOAb: Anti-thyroid peroxidase antibody; TSH: Thyroid-stimulating hormone; UCr: Urinary creatinine; UIC: Urinary iodine concentration; WHO: World Health Organization

Acknowledgements

We thank Mr. Zhenshan Jiao for constructive advice on statistical analysis. 


\section{Funding}

This work was supported by the Chinese National Natural Science key project foundation (Grant 81330064) and the Tianjin Science and Technology Committee project foundation (Grant 14ZCZDSY00022)

\section{Availability of data and materials}

The datasets analysed during the current study are available from the corresponding author on reasonable request.

\section{Authors' contributions}

$\mathrm{KLW}$ and JZ wrote the manuscript. JZ and $M Z$ were involved in the design and conduction of the study. KLW, FAL, HW, LD, YXL, SZ, and $M Z$ were involved in collecting materials. LXL measured UIC in the lab. MZ and WQZ were involved in data interpretation and manuscript writing.

All authors read and approved the final manuscript.

\section{Competing interests}

The authors declare that they have no competing interests.

\section{Consent for publication}

Not applicable.

\section{Ethics approval and consent to participate}

Ethical approval for this study was obtained from the Ethics Committee of Tianjin Medical University General Hospital, Tianjin China (No. IRB-2013-095-01). Informed consent was obtained from all participants.

\section{Author details}

'Department of Endocrinology and Metabolism, Tianjin Medical University General Hospital, No154 Anshan Road, Heping District, Tianjin 300052, China. ${ }^{2}$ Department of Surgery, Tianjin Medical University General Hospital, No154 Anshan Road, Heping District, Tianjin 300052, China. ${ }^{3}$ Department of Nutrition and Food Hygiene, School of Public Health, Tianjin Medical University, Tianjin 300070, China. ${ }^{4}$ Key Lab of Hormone and Development, Institute of Endocrinology, Tianjin Medical University, Tianjin 300070, China. ${ }^{5}$ Project Office, Tianjin Women's and Children's Health Center, No96 Guizhou Road, Heping District, Tianjin 300070, China.

Received: 3 August 2016 Accepted: 15 February 2017

\section{Published online: 17 February 2017}

\section{References}

1. Ashoor G, Maiz N, Rotas M, Jawdat F, Nicolaides KH. Maternal thyroid function at 11 to 13 weeks of gestation and subsequent fetal death. Thyroid. 2010;20:989-93.

2. Liu H, Shan Z, Li C, Mao J, Xie X, Wang W, Fan C, Wang H, Zhang H, Han C, Wang X, Liu U, Fan Y, Bao S, Teng W. Maternal subclinical hypothyroidism, thyroid autoimmunity, and the risk of miscarriage: a prospective cohort study. Thyroid. 2014;24:1642-9.

3. Negro R, Schwartz A, Gismondi R, Tinelli A, Mangieri T, Stagnaro-Green A. Increased pregnancy loss rate in thyroid antibody negative women with TSH levels between 2.5 and 5.0 in the first trimester of pregnancy. J Clin Endocrinol Metab. 2010:95:E44-8.

4. Karakosta P, Alegakis D, Georgiou V, Roumeliotaki T, Fthenou E, Vassilaki M, Boumpas D, Castanas E, Kogevinas M, Chatzi L. Thyroid dysfunction and autoantibodies in early pregnancy are associated with increased risk of gestational diabetes and adverse birth outcomes. J Clin Endocrinol Metab. 2012;97:4464-72

5. Lazarus JH. Thyroid function in pregnancy. Br Med Bull. 2011:97:137-48.

6. Su PY, Huang K, Hao JH, Xu YQ, Yan SQ, Li T, Xu YH, Tao FB. Maternal thyroid function in the first twenty weeks of pregnancy and subsequent fetal and infant development: a prospective population-based cohort study in China. J Clin Endocrinol Metab. 2011;96:3234-41.

7. Lazarus J, Brown RS, Daumerie C, Hubalewska-Dydejczyk A, Negro R, Vaidya B. 2014 European thyroid association guidelines for the management of subclinical hypothyroidism in pregnancy and in children. Eur Thyroid J. 2014;3:76-94.

8. Shi X, Han C, Li C, Mao J, Wang W, Xie X, Li C, Xu B, Meng T, Du J, et al. Optimal and safe upper limits of iodine intake for early pregnancy in iodine-sufficient regions: a cross-sectional study of 7190 pregnant women in China. J Clin Endocrinol Metab. 2015;100:1630-8.
9. Teng W, Shan Z, Teng X, Guan H, Li Y, Teng D, Jin Y, Yu X, Fan C, Chong W, et al. Effect of iodine intake on thyroid diseases in China. N Engl J Med. 2006;354:2783-93.

10. Yan YQ, Chen ZP, Yang XM, Liu H, Zhang JX, Zhong W, Yao W, Zhao JK, Zhang ZZ, Hua JL, et al. Attention to the hiding iodine deficiency in pregnant and lactating women after universal salt iodization: A multicommunity study in China. J Endocrinol Invest. 2005;28:547-53.

11. Mao G, Ding G, Lou X, Zhang R, Zheng P, Mo Z, Wang X, Zhu W, Zhou J, Gu F. Survey of iodine nutritional status in 2011, Zhejiang, China. Asia Pac J Clin Nutr. 2015;24:234-44.

12. Su X, Liu S, Shen H, Zhang S, Wei H, Yu J, Ye Y, Li Y, Liu Y, Zhang Z. National iodine deficiency disorder surveillance: a sum up of data in 2005 and an analysis. Chin J Endem. 2007;26:67-9.

13. Teng W, Duan T, Ning G. Guidelines for the diagnosis and management of thyroid diseases during pregnancy and postpartum. Chin J Endocrinol Metab. 2012;28:356

14. Organization WH. Assessment of iodine deficiency disorders and monitoring their elimination: a guide for programme managers. 2007.

15. Brunn J, Block U, Ruf G, Bos I, Kunze WP, Scriba PC. Volumetric analysis of thyroid lobes by real-time ultrasound (author's transl). Dtsch Med Wochenschr. 1981;106:1338-40. in German.

16. Stagnaro-Green A, Abalovich M, Alexander E, Azizi F, Mestman J, Negro R, Nixon A, Pearce EN, Soldin OP, Sullivan S, et al. Guidelines of the American Thyroid Association for the diagnosis and management of thyroid disease during pregnancy and postpartum. Thyroid. 2011;21:1081-125.

17. Glinoer D. The importance of iodine nutrition during pregnancy. Public Health Nutr. 2007;10:1542-6.

18. Zimmermann MB, Boelaert K. lodine deficiency and thyroid disorders. Lancet Diabetes Endocrinol. 2015;3:286-95.

19. Trumpff C, De Schepper J, Tafforeau J, Van Oyen H, Vanderfaeillie J, Vandevijvere S. Mild iodine deficiency in pregnancy in Europe and its consequences for cognitive and psychomotor development of children: a review. J Trace Elem Med Biol. 2013:27:174-83.

20. Zimmermann MB. The role of iodine in human growth and development. Semin Cell Dev Biol. 2011;22:645-52.

21. Sang Z, Wei W, Zhao N, Zhang G, Chen W, Liu H, Shen J, Liu J, Yan Y, Zhang $W$. Thyroid dysfunction during late gestation is associated with excessive iodine intake in pregnant women. J Clin Endocrinol Metab. 2012; 97:E1363-9.

22. Zimmermann MB, Andersson M. Assessment of iodine nutrition in populations: past, present, and future. Nutr Rev. 2012;70:553-70.

23. Yang J, Zheng H, Li X, Zhu L, Hao Z, Chen G, Liu Y, Wang Y. Assessment of iodine status and associated factors in vulnerable populations in Henan Province, China, in 2012. Asia Pac J Clin Nutr. 2014:23:626-33.

24. Zhang H, Lv S, Mu Z, Li W, Zhang X, Wang Y, Rutherford S. lodised salt contribution to iodine nutrition status of pregnant and lactating women. $\mathrm{Br}$ J Nutr. 2015;114:126-33.

25. Chen W, Hou C, Zhu Y, Li D, Gu J, Liu Z, Chen Z. An evaluation of the effectiveness of implementation of national food safety standard for "lodine Concentratin in Edible Salt"(GB 26878-2011). Chin J Endemiol. 2014:33:407-10.

26. Wolff J, Chaikoff I, Goldberg R, Meier J. The Temporary Nature of The Inhibitory Action of Excess lodide on Organic lodine Synthesis in the Normal Thyroid 1. Endocrinology. 1949:45:504-13.

27. Leung AM, Braverman LE. Consequences of excess iodine. Nat Rev Endocrinol. 2014:10:136-42.

28. Premawardhana L, Parkes A, John R, Harris B, Lazarus J. Thyroid peroxidase antibodies in early pregnancy: utility for prediction of postpartum thyroid dysfunction and implications for screening. Thyroid. 2004;14:610-5.

29. Rago T, Chiovato L, Grasso L, Pinchera A, Vitti P. Thyroid ultrasonography as a too for detecting thyroid autoimmune diseases and predicting thyroid dysfunction in apparently healthy subjects. J Endocrinol Investig. 2001:24:763-9.

30. Wasniewska M, Salerno M, Cassio A, Corrias A, Aversa T, Zirilli G, Capalbo D, Bal M, Mussa A, De Luca F. Prospective evaluation of the natural course of idiopathic subclinical hypothyroidism in childhood and adolescence. Eur J Endocrinol. 2009;160:417-21

31. Cerbone M, Agretti P, De Marco G, Improda N, Pignata C, Santamaria F, Tonacchera M, Salerno M. Non-autoimmune subclinical hypothyroidism due to a mutation in TSH receptor: report on two brothers. Ital J Pediatr. 2013:39:5.

32. Laurberg P, Knudsen N, Andersen S, Carle A, Pedersen IB, Karmisholt Thyroid function and obesity. Eur Thyroid J. 2012;1:159-67. 